\title{
Reconsiderations on the Equivalence of Convergence between Mann and Ishikawa Iterations for Asymptotically Pseudocontractive Mappings
}

\author{
Haizhen Sun and Zhiqun Xue \\ Department of Mathematics and Physics, Shijiazhuang Tiedao University, Shijiazhuang 050043, China \\ Correspondence should be addressed to Zhiqun Xue; xuezhiqun@126.com
}

Received 28 December 2012; Accepted 11 June 2013

Academic Editor: D. R. Sahu

Copyright (C) 2013 H. Sun and Z. Xue. This is an open access article distributed under the Creative Commons Attribution License, which permits unrestricted use, distribution, and reproduction in any medium, provided the original work is properly cited.

Our aim in this paper is to illustrate that the proof of main theorem of Rhoades and Şoltuz (2003) concerning the equivalence between the convergences of Ishikawa and Mann iterations for uniformly L-Lipschitzian asymptotically pseudocontractive maps is incorrect and to provide its correct version.

\section{Introduction and Preliminary}

In 2003, Rhoades and Şoltuz [1] proved the equivalence between convergences of Ishikawa and Mann iterations for an asymptotically pseudocontractive map. This result provided significant improvements of recent some important results. Their result is as follows.

Theorem R-S (see [1, Theorem 8]). Let B be a closed convex subset of an arbitrary Banach space $X$ and $\left(x_{n}\right)_{n}$ and $\left(u_{n}\right)_{n}$ defined by (3) and (4) with $\left(\alpha_{n}\right)_{n}$ and $\left(\beta_{n}\right)_{n}$ satisfying (5). Let T be an asymptotically pseudocontractive and Lipschitzian map with $L \geq 1$ selfmap of $B$. Let $x^{*}$ be the fixed point of $T$. If $u_{0}=x_{0} \in B$, the following two assertions are equivalent:

(i) Mann type iteration (3) converges to $x^{*} \in F(T)$,

(ii) Ishikawa iteration (4) converges to $x^{*} \in F(T)$.

However, after careful reading of the paper of Rhoades and Şoltuz [1], we find that there exists a serious gap in the proof of Theorem 8 of [1], which happens to be main theorem of the paper. Note: in the proof of Theorem 8 of [1] the following mistakes occurred. "Using (6) with $x:=x_{n+1}, y:=$ $u_{n+1}$ " in line 19 of page 684 cannot obtain

$$
\begin{aligned}
& \|\left(1+\alpha_{n}^{2}\right)\left(x_{n+1}-u_{n+1}\right) \\
& \quad+\alpha_{n}\left(\left(\alpha_{n} k_{n} I-T^{n}\right) x_{n+1}-\left(\alpha_{n} k_{n} I-T^{n}\right) u_{n+1}\right) \| \\
& \quad \geq\left(1+\alpha_{n}^{2}\right)\left\|x_{n+1}-u_{n+1}\right\| .
\end{aligned}
$$

The reason is that the following conditions are not equivalent:

(a1) $T$ is asymptotically pseudocontractive map,

(a2) $\|x-y\| \leq\left\|x-y+r\left(\left(\alpha_{n} k_{n} I-T^{n}\right) x-\left(\alpha_{n} k_{n} I-T^{n}\right) y\right)\right\|$, where $\alpha_{n}$ and $k_{n}$ are from (1).

The aim of this paper is for us to provide its correct version. For this, we need the following definitions and lemmas.

Throughout this paper, suppose that $E$ is an arbitrary real Banach space and $D$ is a nonempty closed convex subset of $E$. Let $J$ denote the normalized duality mapping from $E$ to $2^{E^{*}}$ defined by

$$
J(x)=\left\{f \in E^{*}:\langle x, f\rangle=\|x\|^{2}=\|f\|^{2}\right\}, \quad \forall x \in E,
$$

where $E^{*},\langle\cdot, \cdot\rangle$, and $j$ denote the dual space of $E$, the generalized duality pairing, and the single-valued normalized duality mapping, respectively.

Definition 1 (see [1]). Let $T: D \rightarrow D$ be a mapping.

$T$ is called uniformly $L$-Lipschitz if there is a constant $L>$ 0 such that, for all $x, y \in D$,

$$
\left\|T^{n} x-T^{n} y\right\| \leq L\|x-y\|, \quad \forall n \geq 1 .
$$


$T$ is called asymptotically nonexpansive with a sequence $\left\{k_{n}\right\} \subset[1,+\infty)$ and $\lim _{n \rightarrow \infty} k_{n}=1$ if for each $x, y \in D$ such that

$$
\left\|T^{n} x-T^{n} y\right\| \leq k_{n}\|x-y\|, \quad \forall n \geq 1 .
$$

$T$ is called asymptotically pseudocontractive map with a sequence $\left\{k_{n}\right\} \subset[1,+\infty)$ and $\lim _{n \rightarrow \infty} k_{n}=1$ if, for each $x, y \in D$, there exists $j(x-y) \in J(x-y)$ such that

$$
\left\langle T^{n} x-T^{n} y, j(x-y)\right\rangle \leq k_{n}\|x-y\|^{2}, \quad \forall n \geq 1 .
$$

Obviously, an asymptotically nonexpansive mapping is both asymptotically pseudocontractive and uniformly $L$ Lipschitz. Conversely, it is not true in general.

Definition 2 (see [2]). For arbitrary given $u_{1}, x_{1} \in D$, the sequences $\left\{u_{n}\right\}_{n=1}^{\infty},\left\{x_{n}\right\}_{n=1}^{\infty} \subset D$ defined by

$$
\begin{array}{cc}
u_{n+1}=\left(1-a_{n}\right) u_{n}+a_{n} T^{n} u_{n}, & n \geq 1, \\
y_{n}=\left(1-b_{n}\right) x_{n}+b_{n} T^{n} x_{n}, & n \geq 1, \\
x_{n+1}=\left(1-a_{n}\right) x_{n}+a_{n} T^{n} y_{n}, & n \geq 1
\end{array}
$$

are called modified Mann and Ishikawa iterations, respectively, where $\left\{a_{n}\right\},\left\{b_{n}\right\}$ are two real sequences of $[0,1]$ and satisfy some conditions.

Lemma 3 (see [2]). Let $E$ be a real Banach space and $J: E \rightarrow$ $2^{E^{*}}$ be a normalized duality mapping. Then

$$
\|x+y\|^{2} \leq\|x\|^{2}+2\langle y, j(x+y)\rangle,
$$

for all $x, y \in E$ and $j(x+y) \in J(x+y)$.

Lemma 4 (see [3]). Let $\Phi:[0,+\infty) \rightarrow[0,+\infty)$ be a strictly increasing and continuous function with $\Phi(0)=0$, and let $\left\{\delta_{n}\right\}_{n=0}^{\infty},\left\{\lambda_{n}\right\}_{n=0}^{\infty}$, and $\left\{\gamma_{n}\right\}_{n=0}^{\infty}$ be three nonnegative real sequences satisfying the following inequality:

$$
\delta_{n+1}^{2} \leq \delta_{n}^{2}-\lambda_{n} \Phi\left(\delta_{n+1}\right)+\gamma_{n}, \quad n \geq 0,
$$

where $\lambda_{n} \in[0,1]$ with $\sum_{n=0}^{\infty} \lambda_{n}=\infty, \gamma_{n}=o\left(\lambda_{n}\right)$. Then $\delta_{n} \rightarrow$ 0 as $n \rightarrow \infty$.

\section{Main Results}

Now we prove the following theorem which is the main result of this paper.

Theorem 5. Let $E$ be a real Banach space, $D$ be a nonempty closed convex subset of $E$, and $T: D \rightarrow D$ be a uniformly L-Lipschitz asymptotically pseudocontractive mapping with a sequence $\left\{k_{n}\right\} \subset[1,+\infty)$ such that $\lim _{n \rightarrow \infty} k_{n}=1$. Let $\left\{a_{n}\right\},\left\{b_{n}\right\}$ be two real numbers sequences in $[0,1]$ and satisfy the conditions (i) $a_{n}, b_{n} \rightarrow 0$ as $n \rightarrow \infty$; (ii) $\sum_{n=1}^{\infty} a_{n}=\infty$. For some $u_{1}, x_{1} \in D$, let $\left\{u_{n}\right\}$ and $\left\{x_{n}\right\}$ be modified Mann and Ishikawa iterative sequences defined by (6) and (7), respectively. If $F(T)=\{x \in D: F x=x\} \neq \emptyset, q \in F(T)$, and there exists a strictly increasing continuous function $\Phi:[0,+\infty) \rightarrow$ $[0,+\infty)$ with $\Phi(0)=0$ such that

$$
\begin{aligned}
&\left\langle T^{n} x_{n+1}-T^{n} u_{n+1}, j\left(x_{n+1}-u_{n+1}\right)\right\rangle \\
& \leq k_{n}\left\|x_{n+1}-u_{n+1}\right\|^{2}-\Phi\left(\left\|x_{n+1}-u_{n+1}\right\|\right), \\
& \forall n \geq 1,
\end{aligned}
$$

where $j\left(x_{n+1}-u_{n+1}\right) \in J\left(x_{n+1}-u_{n+1}\right)$, then the following two assertions are equivalent:

(1-1) the modified Mann iteration (6) converges strongly to the fixed point $q$ of $T$;

(1-2) the modified Ishikawa iteration (7) converges strongly to the fixed point $q$ of $T$.

Proof. We only need to prove (1-1) $\Rightarrow(1-2)$, that is, $\left\|u_{n}-q\right\| \rightarrow$ 0 as $n \rightarrow \infty \Rightarrow\left\|x_{n}-q\right\| \rightarrow 0$ as $n \rightarrow \infty$. Without loss of generality, $\left\|u_{n}-q\right\| \leq 1$. Since $T: D \rightarrow D$ is a uniformly $L$-Lipschitz, then $\left\|T^{n} x-T^{n} y\right\| \leq L\|x-y\|$.

Step 1 . For any $n \geq 0,\left\{x_{n}\right\}$ is bounded.

Set $u_{n}=q$, for all $n \geq 1, \sup \left\{k_{n}: n \geq 0\right\}=k$, then $(*)$ :

$$
\left\langle T^{n} x_{n+1}-q, j\left(x_{n+1}-q\right)\right\rangle \leq k\left\|x_{n+1}-q\right\|^{2}-\Phi\left(\left\|x_{n+1}-q\right\|\right),
$$

$\forall n \geq 0$.

And there exists $x_{1} \in D$ and $x_{1} \neq T x_{1}$ such that $r_{0}=(k+$ $L) \cdot\left\|x_{1}-q\right\|^{2} \in R(\Phi)$. Indeed, if $\Phi(r) \rightarrow+\infty$ as $r \rightarrow+\infty$, then, $r_{0} \in R(\Phi)$; if $\sup \{\Phi(r): r \in[0,+\infty)\}=r_{1}<+\infty$ with $r_{1}<r_{0}$, then, for $q \in D$, there exists a sequence $\left\{\xi_{n}\right\} \subset D$ such that $\xi_{n} \rightarrow q$ as $n \rightarrow \infty$ with $\xi_{n} \neq q$. Hence there exists a natural number $n_{0}$ such that $(k+L)\left\|\xi_{n}-q\right\|^{2}<r_{1} / 2$ for $n \geq n_{0}$, and then we redefine $x_{1}=\xi_{n_{0}}$ and $(k+L)\left\|x_{1}-q\right\|^{2} \in R(\Phi)$.

Set $R=\Phi^{-1}\left(r_{0}\right)$, and then, from (*), we obtain that

$$
\left\|x_{1}-q\right\| \leq R
$$

Denote $B_{1}=\{x \in D:\|x-q\| \leq R\}, B_{2}=\{x \in D$ : $\|x-q\| \leq 2 R\}$. Next, we want to prove that $x_{n} \in B_{1}$. If $n=1$, then $x_{1} \in B_{1}$. Now assume that it holds for some $n$; that is, $x_{n} \in B_{1}$. We prove that $x_{n+1} \in B_{1}$. Suppose that it is not the case, and then $\left\|x_{n+1}-q\right\|>R$. Now denote

$$
\tau_{0}=\min \left\{\frac{1}{1+2 L}, \frac{\Phi(R)}{16 R^{2}(1+L)(1+2 L)}\right\} \text {. }
$$

Because $a_{n}, b_{n}, k_{n}-1 \rightarrow 0$ as $n \rightarrow \infty$, without loss of generality, let $0 \leq a_{n}, b_{n}, k_{n}-1 \leq \tau_{0}$ for any $n \geq 1$. So we have

$$
\begin{aligned}
\left\|y_{n}-q\right\| & \leq\left(1-b_{n}\right)\left\|x_{n}-q\right\|+b_{n}\left\|T^{n} x_{n}-T^{n} q\right\| \\
& \leq\left(1-b_{n}+b_{n} L\right)\left\|x_{n}-q\right\| \\
& \leq\left(1+b_{n}+b_{n} L\right) R \\
& \leq 2 R,
\end{aligned}
$$




$$
\begin{aligned}
\left\|x_{n+1}-q\right\| \leq & \left(1-a_{n}\right)\left\|x_{n}-q\right\|+a_{n}\left\|T^{n} y_{n}-T^{n} q\right\| \\
\leq & \left(1-a_{n}\right)\left\|x_{n}-q\right\|+a_{n} L\left\|y_{n}-q\right\| \\
\leq & \left(1+a_{n}\right)\left\|x_{n}-q\right\| \\
& +a_{n} L\left(1+b_{n}+b_{n} L\right)\left\|x_{n}-q\right\| \\
\leq & \left(1+a_{n}\right)\left\|x_{n}-q\right\|+2 a_{n} L\left\|x_{n}-q\right\| \\
\leq & 2 R,
\end{aligned}
$$

$$
\left\|x_{n+1}-y_{n}\right\| \leq a_{n}\left\|x_{n}-T^{n} y_{n}\right\|+b_{n}\left\|x_{n}-T^{n} x_{n}\right\|
$$$$
\leq a_{n}\left(\left\|x_{n}-q\right\|+\left\|T^{n} y_{n}-T^{n} q\right\|\right)
$$$$
+b_{n}\left(\left\|x_{n}-q\right\|+\left\|T^{n} x_{n}-T^{n} q\right\|\right)
$$$$
\leq a_{n}\left(\left\|x_{n}-q\right\|+L\left\|y_{n}-q\right\|\right)
$$$$
+b_{n}(1+L)\left\|x_{n}-q\right\|
$$$$
\leq a_{n}(1+2 L) R+b_{n}(1+L) R
$$$$
\leq\left(a_{n}+b_{n}\right)(1+2 L) R
$$$$
\leq \frac{\Phi(R)}{8 R(1+L)},
$$

$$
\begin{aligned}
\leq & \left\|x_{n}-q\right\|^{2}+\frac{2 a_{n}}{1-2 k_{n} a_{n}} \\
\times & {\left[\left[\left(k_{n}-1\right)+\frac{a_{n}}{2}\right]\left\|x_{n}-q\right\|^{2}\right.} \\
& \quad-\Phi\left(\left\|x_{n+1}-q\right\|\right) \\
& \left.\quad+\left\|T^{n} x_{n+1}-T^{n} y_{n}\right\| \cdot\left\|x_{n+1}-q\right\|\right] \\
\leq & \left\|x_{n}-q\right\|^{2}+\frac{2 a_{n}}{1-2 k_{n} a_{n}} \\
& \times\left[\frac{\Phi(R) R^{2}}{8 R^{2}(1+L)(1+2 L)}-\Phi(R)+\frac{\Phi(R) 2 R}{8 R}\right] \\
\leq & \left\|x_{n}-q\right\|^{2}+\frac{2 a_{n}}{1-2 k_{n} a_{n}} \\
& \times\left[\frac{\Phi(R)}{4}-\Phi(R)+\frac{\Phi(R)}{4}\right] \\
\leq & R^{2}-\frac{a_{n}}{1-2 k_{n} a_{n}} \Phi(R) \\
\leq & R^{2},
\end{aligned}
$$

so

$$
\left\|T^{n} x_{n+1}-T^{n} y_{n}\right\| \leq L \frac{\Phi(R)}{8 R(1+L)}<\frac{\Phi(R)}{8 R} .
$$

Using Lemma 3 and the above formula, we obtain

$$
\begin{aligned}
\| x_{n+1} & -q \|^{2} \\
= & \left\|\left(1-a_{n}\right)\left(x_{n}-q\right)+a_{n}\left(T^{n} y_{n}-q\right)\right\|^{2} \\
\leq & \left(1-a_{n}\right)^{2}\left\|x_{n}-q\right\|^{2} \\
& +2 a_{n}\left\langle T^{n} y_{n}-q, j\left(x_{n+1}-q\right)\right\rangle \\
= & \left(1-a_{n}\right)^{2}\left\|x_{n}-q\right\|^{2} \\
& +2 a_{n}\left\langle T^{n} x_{n+1}-q-T^{n} x_{n+1}+T^{n} y_{n}, j\left(x_{n+1}-q\right)\right\rangle \\
\leq & \left(1-a_{n}\right)^{2}\left\|x_{n}-q\right\|^{2} \\
& +2 a_{n}\left[k_{n}\left\|x_{n+1}-q\right\|^{2}-\Phi\left(\left\|x_{n+1}-q\right\|\right)\right] \\
& +2 a_{n}\left\|T^{n} x_{n+1}-T^{n} y_{n}\right\| \cdot\left\|x_{n+1}-q\right\| .
\end{aligned}
$$

Since $2 k_{n} a_{n} \rightarrow 0$ as $n \rightarrow \infty$, without loss of generality, let $1-2 k_{n} a_{n}>0$. Then (17) implies that

$$
\begin{aligned}
\left\|x_{n+1}-q\right\|^{2} \leq & \frac{\left(1-a_{n}\right)^{2}}{1-2 k_{n} a_{n}}\left\|x_{n}-q\right\|^{2} \\
& -\frac{2 a_{n}}{1-2 k_{n} a_{n}} \Phi\left(\left\|x_{n+1}-q\right\|\right) \\
& +\frac{2 a_{n}}{1-2 k_{n} a_{n}}\left\|T^{n} x_{n+1}-T^{n} y_{n}\right\| \cdot\left\|x_{n+1}-q\right\|
\end{aligned}
$$

and this is a contradiction. Hence $x_{n+1} \in B_{1}$; that is, $\left\{x_{n}\right\}$ is a bounded sequence.

Step 2. We show that $\left\|x_{n}-q\right\| \rightarrow 0$ as $n \rightarrow \infty$.

By Step 1, we obtain that $\left\{\left\|x_{n}-u_{n}\right\|\right\}$ is a bounded sequence, and denote $M=\sup _{n}\left\{\left\|x_{n}-u_{n}\right\|\right\}$. Applying (6), (7), and Lemma 3, we have

$$
\begin{aligned}
\left\|x_{n+1}-u_{n+1}\right\|^{2}= & \left\|\left(1-a_{n}\right)\left(x_{n}-u_{n}\right)+a_{n}\left(T^{n} y_{n}-T^{n} u_{n}\right)\right\|^{2} \\
\leq & \left(1-a_{n}\right)^{2}\left\|x_{n}-u_{n}\right\|^{2} \\
& +2 a_{n}\left\langle T^{n} y_{n}-T^{n} u_{n}, j\left(x_{n+1}-u_{n+1}\right)\right\rangle \\
\leq & \left(1-a_{n}\right)^{2}\left\|x_{n}-u_{n}\right\|^{2} \\
& +2 a_{n}\left\langle T^{n} y_{n}-T^{n} x_{n+1}, j\left(x_{n+1}-u_{n+1}\right)\right\rangle \\
& +2 a_{n}\left\langle T^{n} x_{n+1}-T^{n} u_{n+1}, j\left(x_{n+1}-u_{n+1}\right)\right\rangle \\
& +2 a_{n}\left\langle T^{n} u_{n+1}-T^{n} u_{n}, j\left(x_{n+1}-u_{n+1}\right)\right\rangle \\
\leq & \left(1-a_{n}\right)^{2}\left\|x_{n}-u_{n}\right\|^{2} \\
& +2 a_{n} L\left\|y_{n}-x_{n+1}\right\| \cdot\left\|x_{n+1}-u_{n+1}\right\| \\
& +2 a_{n}\left[k_{n}\left\|x_{n+1}-u_{n+1}\right\|^{2}\right. \\
& \left.\quad-\Phi\left(\left\|x_{n+1}-u_{n+1}\right\|\right)\right] \\
& +2 a_{n} L\left\|u_{n+1}-u_{n}\right\| \cdot\left\|x_{n+1}-u_{n+1}\right\| .
\end{aligned}
$$


Observe that

$$
\begin{aligned}
\left\|y_{n}-x_{n+1}\right\|= & \left\|a_{n}\left(x_{n}-T^{n} y_{n}\right)-b_{n}\left(x_{n}-T^{n} x_{n}\right)\right\| \\
\leq & \left(a_{n}+b_{n}+b_{n} L\right)\left\|x_{n}-q\right\|+a_{n} L\left\|y_{n}-q\right\| \\
\leq & \left(a_{n}+b_{n}+b_{n} L\right)\left\|x_{n}-q\right\| \\
& +a_{n} L\left(1+b_{n} L\right)\left\|x_{n}-q\right\| \\
\leq & \left(a_{n}+b_{n}+b_{n} L+a_{n} L+a_{n} b_{n} L^{2}\right)\left\|x_{n}-q\right\| \\
\leq & h_{n}\left(\left\|x_{n}-u_{n}\right\|+\left\|u_{n}-q\right\|\right) \\
\leq & h_{n}\left(\left\|x_{n}-u_{n}\right\|+1\right), \\
\left\|u_{n+1}-u_{n}\right\|= & \left\|a_{n}\left(T^{n} u_{n}-u_{n}\right)\right\| \\
= & a_{n}\left\|T^{n} u_{n}-T^{n} q+q-u_{n}\right\| \\
\leq & a_{n}(1+L)\left\|u_{n}-q\right\| \\
\leq & a_{n}(1+L),
\end{aligned}
$$

where $h_{n}=a_{n}+b_{n}+b_{n} L+a_{n} L+a_{n} b_{n} L^{2} \rightarrow 0$ as $n \rightarrow \infty$.

Substituting (20) and (21) into (19), we obtain

$$
\begin{aligned}
\| x_{n+1} & -u_{n+1} \|^{2} \\
\leq & \left(1-a_{n}\right)^{2}\left\|x_{n}-u_{n}\right\|^{2} \\
& +2 L a_{n} h_{n}\left(\left\|x_{n}-u_{n}\right\|+1\right)\left\|x_{n+1}-u_{n+1}\right\| \\
& +2 a_{n}\left(k_{n}\left\|x_{n+1}-u_{n+1}\right\|^{2}-\Phi\left(\left\|x_{n+1}-u_{n+1}\right\|\right)\right) \\
& +2 a_{n} L a_{n}(1+L)\left\|x_{n+1}-u_{n+1}\right\| \\
\leq & \left(1-a_{n}\right)^{2}\left\|x_{n}-u_{n}\right\|^{2} \\
& +L a_{n} h_{n}\left(\left\|x_{n}-u_{n}\right\|^{2}+2\left\|x_{n+1}-u_{n+1}\right\|^{2}+1\right) \\
& +2 a_{n}\left(k_{n}\left\|x_{n+1}-u_{n+1}\right\|^{2}-\Phi\left(\left\|x_{n+1}-u_{n+1}\right\|\right)\right) \\
& +L(1+L) a_{n}^{2}+L(1+L) a_{n}^{2}\left\|x_{n+1}-u_{n+1}\right\|^{2} .
\end{aligned}
$$

Since $a_{n} \rightarrow 0$ as $n \rightarrow \infty$, without loss of generality, we may assume that

$$
\frac{1}{2}<1-2 L a_{n} h_{n}-2 a_{n} k_{n}-L(1+L) a_{n}^{2}<1,
$$

for any $n \geq 1$. Then, (22) implies that

$$
\begin{aligned}
\| x_{n+1} & -u_{n+1} \|^{2} \\
\leq & \frac{\left(1-a_{n}\right)^{2}+L a_{n} h_{n}}{1-2 L a_{n} h_{n}-2 a_{n} k_{n}-L(1+L) a_{n}^{2}} \\
& \times\left\|x_{n}-u_{n}\right\|^{2}
\end{aligned}
$$

$$
\begin{aligned}
& +\frac{L a_{n} h_{n}+L(1+L) a_{n}^{2}}{1-2 L a_{n} h_{n}-2 a_{n} k_{n}-L(1+L) a_{n}^{2}} \\
& -\frac{2 a_{n}}{1-2 L a_{n} h_{n}-2 a_{n} k_{n}-L(1+L) a_{n}^{2}} \Phi \\
& \times\left(\left\|x_{n+1}-u_{n+1}\right\|\right) \\
& =\left(1+\frac{2 a_{n}\left(k_{n}-1\right)+a_{n}^{2}+3 L a_{n} h_{n}+L(1+L) a_{n}^{2}}{1-2 L a_{n} h_{n}-2 a_{n} k_{n}-L(1+L) a_{n}^{2}}\right) \\
& \times\left\|x_{n}-u_{n}\right\|^{2} \\
& +\frac{L a_{n} h_{n}+L(1+L) a_{n}^{2}}{1-2 L a_{n} h_{n}-2 a_{n} k_{n}-L(1+L) a_{n}^{2}} \\
& +\frac{2 a_{n}}{1-2 L a_{n} h_{n}-2 a_{n} k_{n}-L(1+L) a_{n}^{2}} \Phi \\
& + \\
& +\left[x_{n}-u_{n} \|^{2}\right. \\
& \leq \\
& +\left(1+x_{n+1}-u_{n+1} \|\right) \\
& \left.\times \| a_{n}\left(k_{n}-1\right)+2 a_{n}^{2}+2 L(1+L) a_{n}^{2}+6 L a_{n} h_{n}\right] M^{2} \\
& \left.+2 a_{n}-u_{n} \|^{2}+2 L(1)+2 a_{n}^{2}+2 L(1+L) a_{n}^{2}+6 L a_{n} h_{n}\right) \\
& \left.+u_{n+1} \|\right) \\
& +L) a_{n}^{2}+2 L a_{n} h_{n}-2 a_{n} \Phi\left(\left\|x_{n+1}-u_{n+1}\right\|\right) \\
& +2 L a_{n} h_{n}
\end{aligned}
$$

Let $\delta_{n}=\left\|x_{n}-u_{n}\right\|, \lambda_{n}=2 a_{n}, \gamma_{n}=\left[4 a_{n}\left(k_{n}-1\right)+2(1+L+\right.$ $\left.\left.L^{2}\right) a_{n}^{2}+6 L a_{n} h_{n}\right] M^{2}+2 L(1+L) a_{n}^{2}+2 L a_{n} h_{n}=o\left(\lambda_{n}\right)$. Then (24) leads to

$$
\delta_{n+1}^{2} \leq \delta_{n}^{2}-\lambda_{n} \Phi\left(\delta_{n+1}\right)+\gamma_{n}
$$

By Lemma 4, we obtain $\lim _{n \rightarrow \infty} \delta_{n}=0$. That is, $\left\|x_{n}-u_{n}\right\| \rightarrow$ 0 as $n \rightarrow \infty$. From the inequality $0 \leq\left\|x_{n}-q\right\| \leq\left\|x_{n}-u_{n}\right\|+$ $\left\|u_{n}-q\right\|$, we get $\left\|x_{n}-q\right\| \rightarrow 0$ as $n \rightarrow \infty$. This completes the proof.

Remark 6. The error in the proof of Theorem 8 of [1] has been pointed out and corrected, but it is not easy what the author really wants to obtain the proof of Theorem 8 in [1] at present.

Remark 7. The proof method of Theorem 5 is quite different from that of [1] and others.

\section{Acknowledgments}

This work was supported by Hebei Provincial Natural Science Foundation (Grant no. A2011210033). And authors thank the reviewers for good suggestions and valuable comments of the paper. 


\section{References}

[1] B. E. Rhoades and S. M. Şoltuz, "The equivalence between the convergences of Ishikawa and Mann iterations for an asymptotically pseudocontractive map," Journal of Mathematical Analysis and Applications, vol. 283, no. 2, pp. 681-688, 2003.

[2] S. S. Zhang, "Iterative approximation problem of fixed points for asymptotically nonexpansive mappings in Banach spaces," Acta Mathematicae Applicatae Sinica, vol. 24, no. 2, pp. 236-241, 2001.

[3] C. Moore and B. V. C. Nnoli, "Iterative solution of nonlinear equations involving set-valued uniformly accretive operators," Computers \& Mathematics with Applications, vol. 42, no. 1-2, pp. 131-140, 2001. 


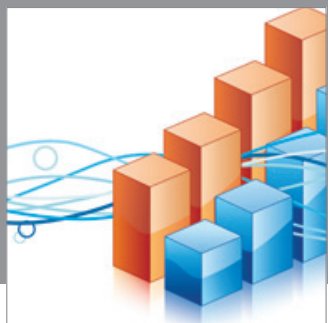

Advances in

Operations Research

mansans

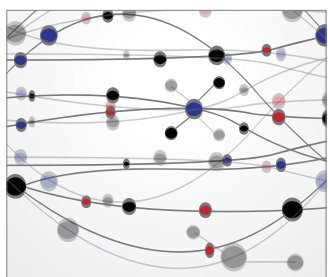

The Scientific World Journal
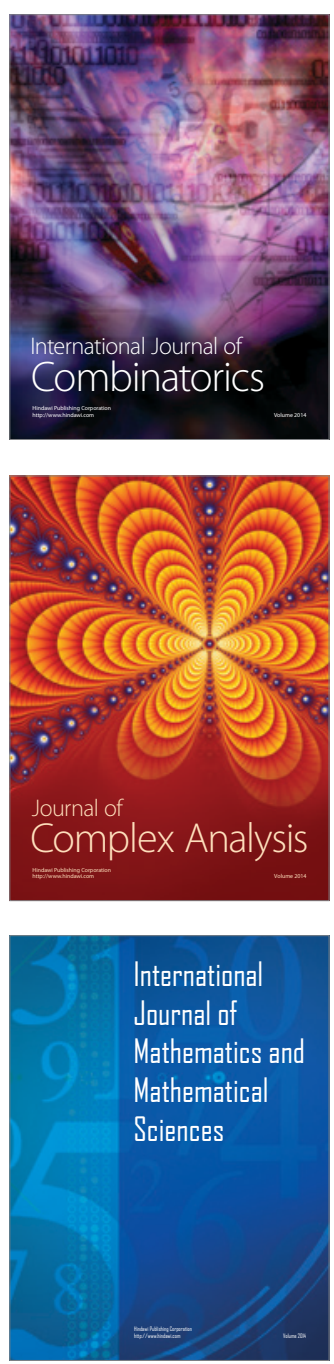
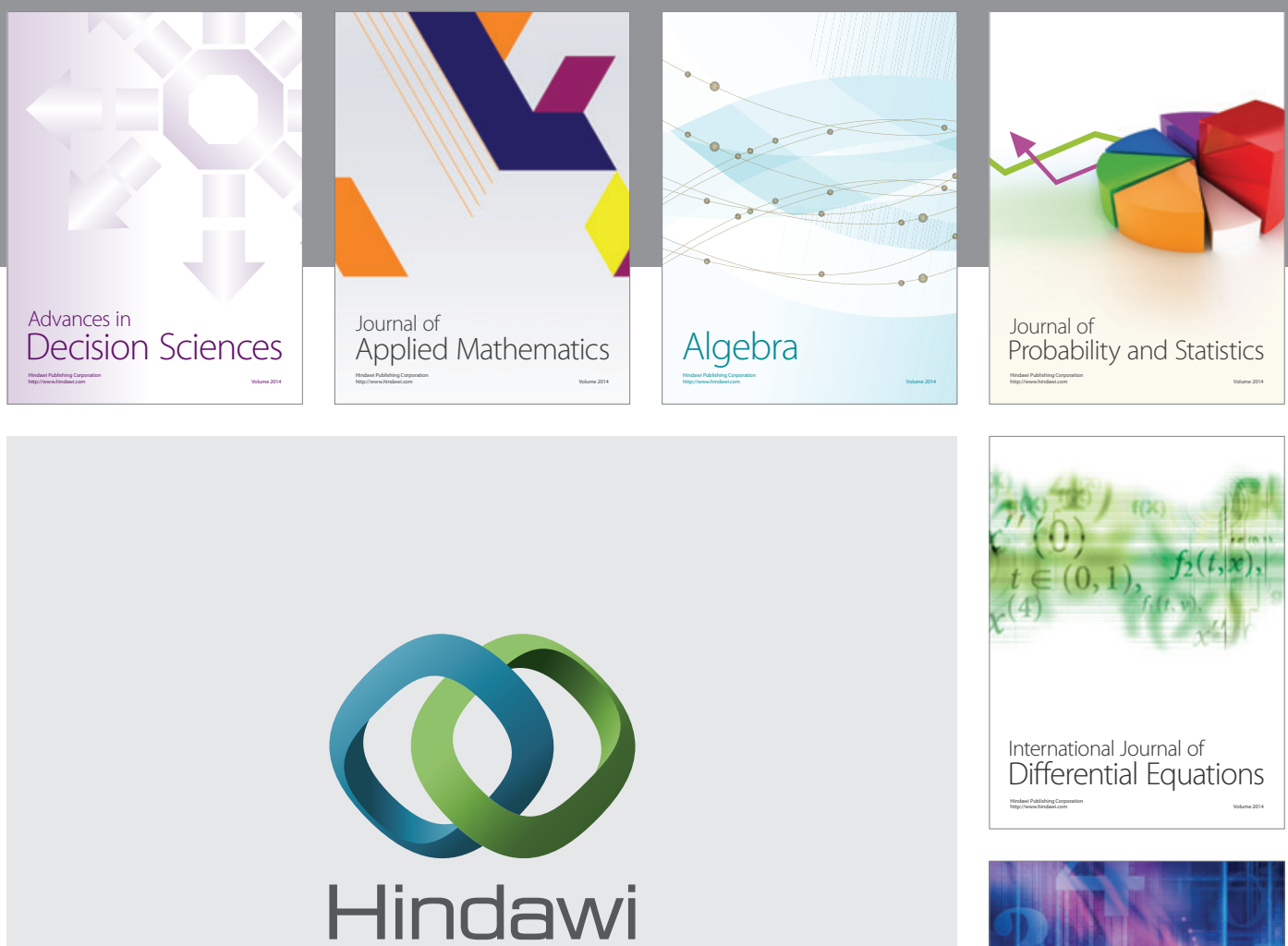

Submit your manuscripts at http://www.hindawi.com
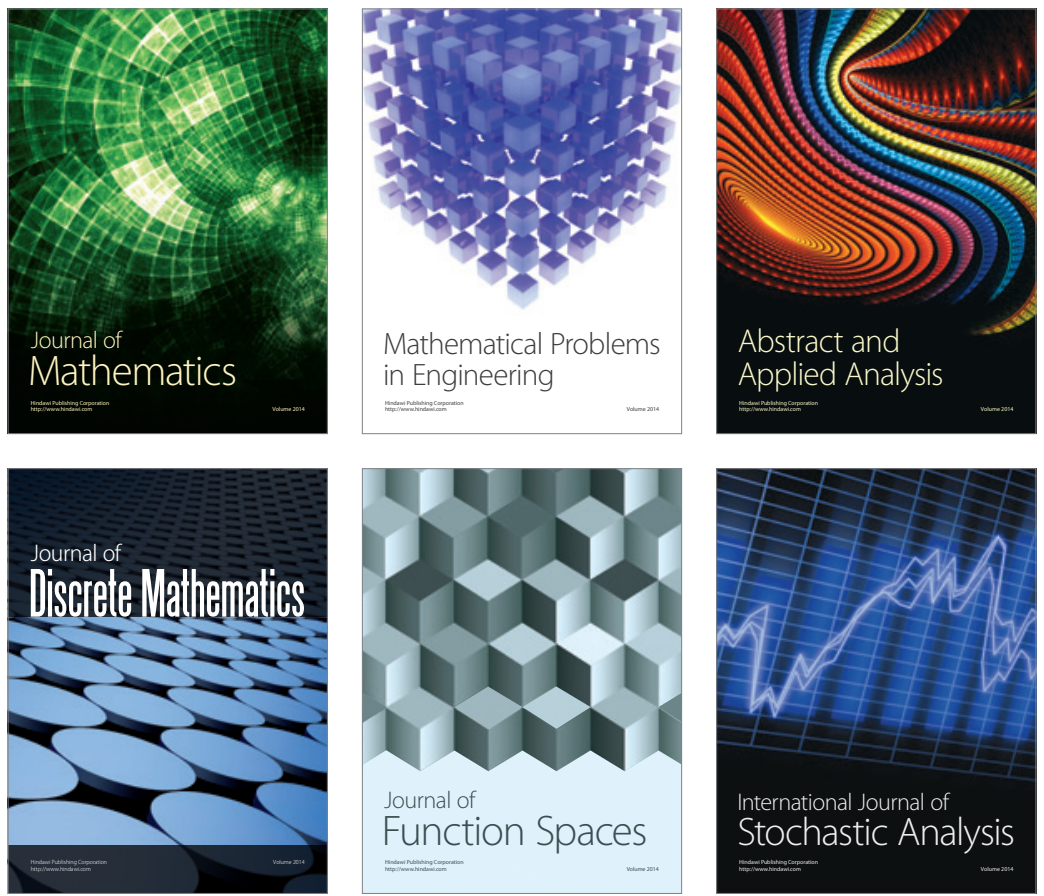

Journal of

Function Spaces

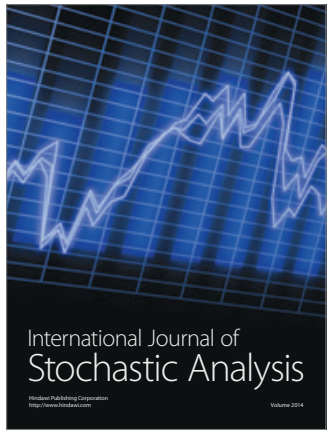

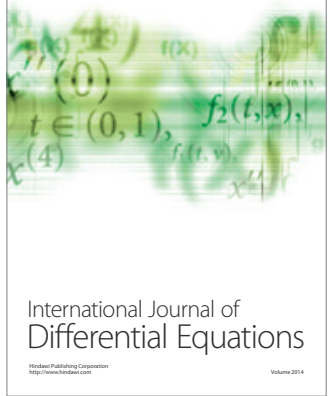
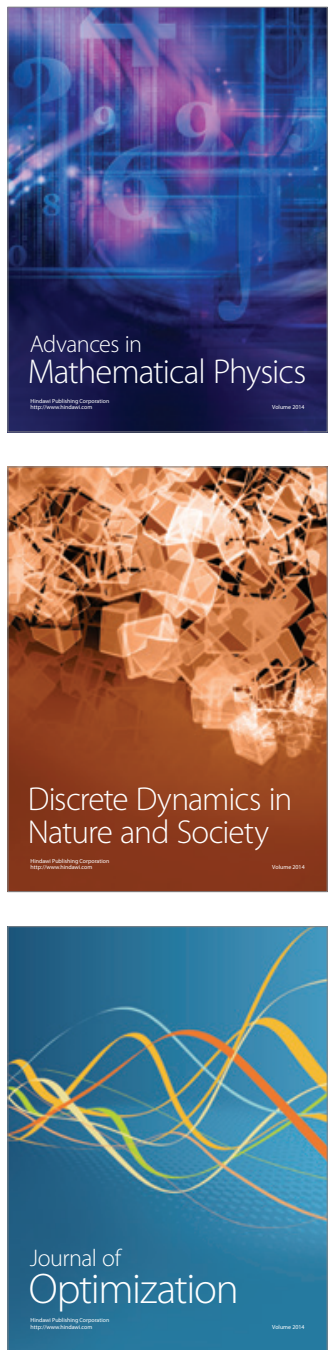